\title{
Violence conjugale, maltraitance et garde des enfants : une démarche de recherche-action favorisant la résolution des controverses entre les acteurs et l'émergence d'une pratique novatrice concerté ${ }^{1}$
}

Geneviève Lessard, Ph.D.

Université Laval

Catherine Flynn, Doctorante

Université de Montréal

Pierre Turcotte, Ph.D.

Université Laval

Lorraine Juneau, B.Serv.soc.

Centre jeunesse de Québec - Institut universitaire

Rhéa Delisle, B.Tr.soc.

Centre de santé et de services sociaux de la Vieille-Capitale

Valérie Meunier, B.Ps.

Groupe d'aide aux personnes impulsives

Marie-France Godin, D.G.

Maison de la famille DVS

RECHERCHES QUALITATIVES - Vol. 31(2), pp. 25-51.

LA RECHERCHE QUALITATIVE AU SERVICE DU CHANGEMENT

ISSN 1715-8702 - http://www.recherche-qualitative.qc.ca/Revue.html

(C) 2012 Association pour la recherche qualitative 
Yennelys Alcedo, Intervenante

Maison pour femmes immigrantes

Annie Dumont, Étudiante à la maîtrise

\title{
Université Laval
}

\begin{abstract}
Résumé
Cet article discute des résultats d'une recherche-action visant à élaborer un processus de résolution des controverses entourant la garde des enfants à la fois exposés à la violence conjugale et victimes de mauvais traitements. S'inspirant de la sociologie de l'innovation comme cadre théorique et de la méthodologie propre aux recherches-actions, la démarche expérimentée avec six milieux de pratique de la région de Québec est exposée et discutée. Les résultats font ressortir les conditions ayant favorisé l'émergence d'une intervention novatrice et plus concertée.

Mots clés

VIOLENCE CONJUGALE, MALTRAITANCE, RECHERCHE-ACTION, CONCERTATION
\end{abstract}

\section{Introduction}

Si la violence conjugale et la maltraitance envers les enfants ont longtemps été considérées, par les praticiens et les chercheurs, comme des réalités distinctes, de plus en plus d'écrits scientifiques montrent qu'elles se présentent souvent de manière concomitante (Appel \& Holden, 1998; Goddard \& Bedi, 2010). Cet article porte sur une recherche-action réalisée en collaboration avec des milieux de pratique concernés par cette double problématique. L'article débute par une synthèse de la problématique étudiée. Les fondements théoriques et méthodologiques du projet sont ensuite présentés. Puis, dans l'objectif de contribuer à une meilleure compréhension du déroulement et des enjeux de cette recherche-action, nous avons choisi d'intégrer la présentation des résultats à leur discussion.

\section{Violence conjugale et maltraitance : enjeux liés à la concertation}

La concomitance de violence conjugale et de maltraitance des enfants est de plus en plus documentée dans les écrits scientifiques (Appel \& Holden, 1998; Goddard \& Bedi, 2010; Lavergne, Chamberland, Laporte, \& Baraldi, 2003; Margolin, Vickerman, Ramos, Serrano, Gordis, Iturralde, Oliver, \& Spies, 2009). À partir de l'analyse de 31 études, Goddard et Bedi (2010) situent entre $40 \%$ et $55 \%$ le taux de prévalence de cette concomitance. Dans ces situations, 
la violence est plus sévère (Shipman, Rossman, \& West, 1999) et peut s'accompagner d'autres problématiques comme la toxicomanie, la pauvreté et les problèmes de santé mentale (Hartley, 2002; Osofsky, 2003).

Dans l'intervention auprès des familles en situation de concomitance, les enjeux liés à la garde des enfants et à la sécurité des victimes s'avèrent particulièrement préoccupants (Lessard, Flynn, Turcotte, Damant, Vézina, Godin, Paradis, Delisle, Alcedo, Juneau, Rock, \& Rondeau-Cantin, 2010; Postmus \& Ortega, 2005). La séparation conjugale est susceptible de créer un contexte propice à la continuation voire même à une hausse de la violence au sein de ces familles (Jaffe, Crooks, \& Poisson, 2003). Les contacts entre les enfants et leur père aux comportements violents peuvent devenir le mécanisme par lequel ce dernier continue ou exacerbe son contrôle envers leur mère (Rinfret-Raynor, Dubé, Drouin, Maillé, \& Harper, 2008). D'où l'importance que les organismes qui supervisent les contacts et les échanges de garde reçoivent une formation plus spécialisée en ce qui a trait aux dynamiques de violence conjugale et familiale (Tutty, 2007). Les démarches judiciaires liées à la garde des enfants peuvent représenter un outil de contrôle sur l'ex-conjointe (Jaffe et al., 2003). Cette dernière risque également d'être revictimisée par le processus judiciaire, ou même accusée de ne pas avoir protégé adéquatement son enfant du contexte de violence (Beeman, Hagemeister, \& Edleson, 1999), accusations susceptibles de jouer contre elle dans les décisions entourant la garde des enfants (Jaffe, Lemmon, \& Poisson, 2005). Dans un tel contexte, il importe de demeurer prudent afin que les modalités de garde ne contribuent pas à compromettre la sécurité des enfants et de leur mère (Lapierre, Lessard, \& Turcotte, 2004).

L'intervention auprès des familles en situation de concomitance représente un défi considérable en raison des difficultés d'arrimage entre les ressources destinées aux agresseurs, aux victimes et aux enfants exposés à la violence ou maltraités. Alors que plusieurs auteurs ont souligné l'importance d'améliorer les relations de collaboration entre ces différents milieux, afin d'offrir aux familles concernées une aide intégrée et cohérente (Douglas \& Walsh, 2010; Potito, Day, Carson, \& O’Leary, 2009), d'autres proposent des programmes d'intervention novateurs (Banks, Hazen, Coben, Wang, \& Griffith, 2009; Malik, Silverman, Wang, \& Janczewski, 2008a; Wendt, 2010).

Parmi l'ensemble des initiatives de concertation recensées, deux ciblent spécifiquement les familles en situation de concomitance : un projet américain et un projet australien. Ces projets se rejoignent quant à leurs objectifs ainsi qu'aux milieux de pratique partenaires. Ils visent à développer des politiques et des procédures d'intervention pour améliorer la sécurité et le bien-être des 
femmes et des enfants confrontés à la violence. Les partenaires impliqués sont principalement les services de protection de l'enfance et les maisons d'hébergement pour femmes violentées. Le projet américain implique également les services du milieu judiciaire. D'autres organismes, comme les organismes pour conjoints aux comportements violents, les services en santé mentale ainsi que les collaborateurs en provenance des communautés autochtones ou des groupes ethniques minoritaires, peuvent également être impliqués (Banks, Dutch, \& Wang, 2008).

Aux États-Unis, des comités de coordination locaux ont été implantés dans six communautés et évalués sur une période de cinq ans (Banks et al., 2009; Malik et al., 2008a). Selon la majorité des participants, bien que leur action n'ait pas entraîné des changements majeurs dans les politiques et les pratiques, le projet a permis de les améliorer dans certains sites (Banks et al., 2009). Les efforts de collaboration semblent coïncider avec une plus grande préoccupation accordée à la sécurité des victimes et à la nécessité des services intégrés (Malik et al., 2008a). En ce qui a trait au projet australien, il a permis d'identifier quatre conditions à mettre en place pour favoriser la collaboration entre les organismes concernés par l'offre de services aux familles en situation de concomitance : 1) créer un contexte favorisant l'émergence d'un sentiment de confiance, 2) identifier des objectifs communs, 3) comprendre les différents rapports de pouvoir entre eux, 4) communiquer avec transparence (Potito et al., 2009).

Ces deux projets de concertation en violence conjugale et en maltraitance présentent trois enjeux importants ayant complexifié l'arrimage entre les acteurs, soit : les divergences de représentations de la problématique, les relations de pouvoir inégales entre les acteurs et les limites temporelles. D'abord, le manque de connaissances communes concernant la problématique de la concomitance et les ressources d'aide ont représenté un obstacle rencontré au sein des initiatives de concertation américaine et australienne. Les ressources partenaires divergent quant à leurs compréhensions et leurs croyances en regard de la violence (Banks et al., 2008; Wendt, 2010), ce qui entraîne des frustrations, des difficultés de communication et des attitudes nuisibles à la collaboration (Wendt, 2010). Pour faire face à ces difficultés, Wendt (2010) propose que les partenaires apprennent à mieux se connaître à travers des activités favorisant le réseautage et la discussion sur la problématique de la concomitance. Pour tendre vers une représentation nouvelle et élargie de la concomitance, le leadership s'avère important afin de mobiliser les partenaires dans un travail de réflexion sur la problématique (Banks et al., 2009; Malik, Ward, \& Janczewski, 2008b). 
L'importance d'égaliser les rapports de pouvoir entre les organismes partenaires est aussi bien documentée (Lessard, Lavergne, Chamberland, Damant, \& Turcotte, 2006; Potito et al., 2009), puisque les rapports inégaux entre ces derniers ressortent comme un obstacle majeur à la collaboration (Banks et al., 2008). Le pouvoir légal et l'importance des ressources financières et matérielles propres aux services judiciaires impliqués dans le projet américain ont placé ces acteurs en position de pouvoir par rapport aux organismes communautaires d'aide en violence conjugale ayant des moyens plus limités (Banks et al., 2008). Bien que les partenaires des services judiciaires aient montré un engagement réel dans le projet et fait des efforts de collaboration avec les différents organismes partenaires, ces derniers percevaient, comparativement aux juges par exemple, plus d'inégalités dans les relations. Ces difficultés se sont d'ailleurs soldées, dans certains sites, par l'interruption ou même la fin du projet (Malik et al., 2008b).

Enfin, les participants au projet américain se sont butés à des limites temporelles dans leurs efforts de collaboration. Le manque de temps s'est avéré particulièrement critique au moment de l'évaluation des besoins, dans la construction des liens de collaboration et dans l'engagement de la communauté (Malik et al., 2008b). De la même façon, pour le projet australien, Potito et ses collaborateurs (2009) soulignent que la création d'un lien de confiance entre les ressources partenaires se tisse graduellement et nécessite beaucoup de temps.

En somme, malgré la complexité de la problématique et les défis associés à la concertation entre les diverses ressources d'aide concernées, les projets novateurs recensés renforcent l'importance d'offrir aux familles en situation de concomitance une aide mieux intégrée et plus cohérente. La présente recherche-action s'inscrit dans cette perspective : elle visait à élaborer une stratégie de concertation novatrice favorisant la résolution des controverses en lien avec la garde des enfants entre les acteurs concernés par l'aide aux familles en situation de concomitance.

\section{Cadre théorique}

La sociologie de l'innovation, développée par Callon et Latour en 1986 (Amblard, Bernoux, Herreros, \& Livian, 1996), constitue un cadre de référence utile pour analyser le processus de résolution des controverses liées à la garde des enfants dans les cas de concomitance. En effet, elle propose une théorisation du processus par lequel des acteurs, qui ancrent leurs pratiques dans des univers culturels différents et qui ont des intérêts distincts de même qu'un accès différent aux ressources et à l'autorité, peuvent réussir à réconcilier leurs intérêts et participer à un projet collectif novateur. L'enjeu majeur consiste à élaborer des ponts menant vers une nouvelle construction à laquelle 
tous auront contribué. Le processus comporte quatre étapes : 1) la situation est analysée de manière à mieux comprendre les acteurs en présence, leurs intérêts respectifs, ce qui les rallie et ce qui les distingue (problématisation); 2) des stratégies sont élaborées afin d'intéresser les acteurs au problème et de sceller les alliances (intéressement); 3) pour chaque groupe d'acteurs, des rôles précis sont définis, négociés et acceptés, en vue d'établir les fondements d'une collaboration (enrôlement) et enfin; 4) des efforts sont faits pour la mobilisation des acteurs impliqués dans l'action (Amblard et al., 1996). À notre connaissance, ce cadre théorique n'a pas été appliqué à la résolution des controverses entourant la garde des enfants en situation de concomitance, ce qui constitue l'originalité de la présente recherche. S'inspirant des conditions favorables à la qualité des actions de concertation identifiées dans la littérature (Bilodeau, Lapierre, \& Marchand, 2003; Potito et al., 2009), la présente recherche permet d'approfondir les connaissances sur l'élaboration et la mise en place d'une pratique novatrice et concertée entre des acteurs aux représentations et aux intérêts divergents.

\section{Méthode}

Les objectifs spécifiques de cette recherche-action étaient : 1) d'identifier les acteurs concernés par la concomitance et d'améliorer la compréhension de leurs contextes de pratique respectifs; 2) de cerner plus précisément les controverses entourant la garde des enfants dans les cas de concomitance; 3) d'élaborer une stratégie novatrice de concertation favorisant la résolution des principales controverses; 4) d'expérimenter et d'évaluer la stratégie élaborée dans l'intervention auprès de quelques familles, en vue d'apporter des réajustements si nécessaire et d'émettre des recommandations pour la pratique et les recherches futures. La recherche-action s'est déroulée en trois principales phases, en cohérence avec les étapes proposées par Lavoie, Marquis et Laurin (2003), soit : la définition du problème et de son contexte, la planification et la réalisation de l'action combinées à une observation continue ainsi qu'à une collecte de données et, finalement, l'évaluation de l'action et la prise de décision.

D'abord, afin de mieux comprendre le problème, son contexte et les groupes d'acteurs concernés, des données ont été recueillies par le biais de groupes focalisés dans chacun des milieux de pratique concernés par la problématique dans la région de Québec ( $n=43$ intervenants). Cette première étape a permis de mieux connaître les acteurs, leurs points de vue et leurs pratiques en ce qui a trait à la garde des enfants en situation de concomitance. Par la suite, deux à trois intervenants de chaque contexte souhaitant poursuivre la démarche ont participé à un groupe de travail intersectoriel (deux journées 
de travail réunissant une vingtaine d'intervenants), dont le mandat était de débattre des controverses et d'identifier quelques pistes de solution favorisant la résolution des controverses. À partir des synthèses de ces rencontres, le comité de coordination du projet a poursuivi le travail d'élaboration de la stratégie retenue. Troisièmement, la stratégie a été expérimentée dans l'intervention auprès de cinq familles et évaluée par un questionnaire maison (échelles de Likert et questions ouvertes) complété par les intervenants participants aux rencontres de discussions cliniques ( $\mathrm{n}=24$ intervenants).

Le comité de coordination a joué un rôle central dans le déroulement de la recherche-action et pour l'atteinte des objectifs. Nous reviendrons sur cet aspect dans la présentation des résultats. Outre les chercheurs universitaires et les étudiants, le comité de coordination est composé de praticiens-chercheurs (Reason \& Bradbury, 2001) représentant chacun des milieux de pratique partenaires du projet, soit : le Centre jeunesse de Québec - Institut universitaire (CJQ-IU), le Centre de santé et de services sociaux de la Vieille-Capitale (CSSS-VC), la Maison d'hébergement pour femmes immigrantes, le Groupe d'aide aux personnes impulsives, la Maison de la famille DVS et le Centre d'amitié autochtone de Québec. Les rencontres du comité ont eu lieu environ tous les deux mois, pour un total de 19 rencontres depuis le début officiel du projet en février 2006, outre les trois rencontres réalisées en 2004 pour la conception du projet.

Les principales sources de données utilisées pour la production du présent article ont été les comptes-rendus (CR) des rencontres du comité de coordination, rédigés par l'auxiliaire de recherche, puis validés ou complétés par les autres membres du comité au début de la rencontre suivante, ainsi que les journaux de bord (JB) complétés après les rencontres du comité par chacun des membres. Ce matériel d'analyse comprend 94 pages de texte à simple interligne (72,5 pages pour les CR et 21,5 pages pour les JB). Pour la rédaction de leur JB, les membres étaient invités à rédiger de courts paragraphes résumant leur point de vue sur: le déroulement des rencontres du comité de coordination (climat et contenus discutés) et le déroulement du projet (étapes, orientations générales, obstacles et éléments facilitants). Les membres ont complété ces JB de février 2006 à août 2008. Par la suite, les JB ont été remplacés par une évaluation systématique à la fin des rencontres du comité reprenant ces mêmes thèmes, ces contenus étant intégrés aux CR. Les citations tirées de ces documents seront identifiées comme suit dans la présentation des résultats : «JB-mois et année» et «CR-mois et année». Ces données qualitatives ont été analysées par la chercheuse principale et les auxiliaires de recherche à l'aide de la méthode d'analyse de contenu thématique (Mayer \& Deslauriers, 2000). L'analyse visait à identifier les facteurs qui ont favorisé le 
processus d'élaboration de la stratégie novatrice, pour chacune des étapes du cadre théorique retenu (problématisation, intéressement, enrôlement, mobilisation). Les concepts de la sociologie de l'innovation ont donc servi de catégories principales structurant l'analyse, autour desquelles se greffent les thèmes émergeant du corpus de données. Les analyses se sont déroulées de façon continue par la discussion, au comité de coordination, des facteurs influençant la démarche, bien que des périodes plus intenses d'analyses aient été réalisées à la fin de chacune des trois phases du projet. Les résultats ont été discutés et validés par les membres du comité de coordination.

Le projet a été approuvé par les comités d'éthique des établissements concernés, soit le comité d'éthique du CSSS-VC et celui du CJQ-IU. Chaque intervenant participant à l'une ou l'autre des phases du projet l'a fait de façon volontaire et a signé un formulaire de consentement.

\section{La démarche expérimentée et ses fondements théoriques et méthodologiques}

Dans le cadre de cette recherche-action, les phases de problématisation, d'intéressement, d'enrôlement et de mobilisation se sont déployées à deux niveaux. D'abord, à plus petite échelle, on observe l'ancrage de ces processus au sein du comité de coordination du projet. La première partie des résultats présentés porte sur les conditions qui se sont avérées essentielles à l'enrôlement des membres du comité de coordination. Dans un deuxième temps, d'autres intervenants ont été impliqués dans la démarche. Les résultats présentés dans les deuxième et troisième sections décrivent les processus d'intéressement, d'enrôlement et de mobilisation des acteurs ne faisant pas partie du comité de coordination. Les obstacles rencontrés ainsi que les stratégies privilégiées pour les contourner sont également discutés.

Les membres du comité de coordination ont joué un rôle clé à la fois pour l'atteinte des objectifs du projet et pour favoriser l'intéressement, l'enrôlement et la mobilisation d'autres acteurs dans le projet. La Figure 1 illustre qu'il s'agit d'une démarche cyclique, ce qui est d'ailleurs conforme aux écrits méthodologiques sur la recherche-action (Lavoie et al., 2003). La continuité entre les étapes s'avère également une caractéristique centrale du processus. En effet, bien que la problématisation ait occupé une plus grande importance en début de projet alors que la fin du projet se caractérise davantage par la mobilisation dans l'action, ces diverses étapes demeurent étroitement interreliées. 


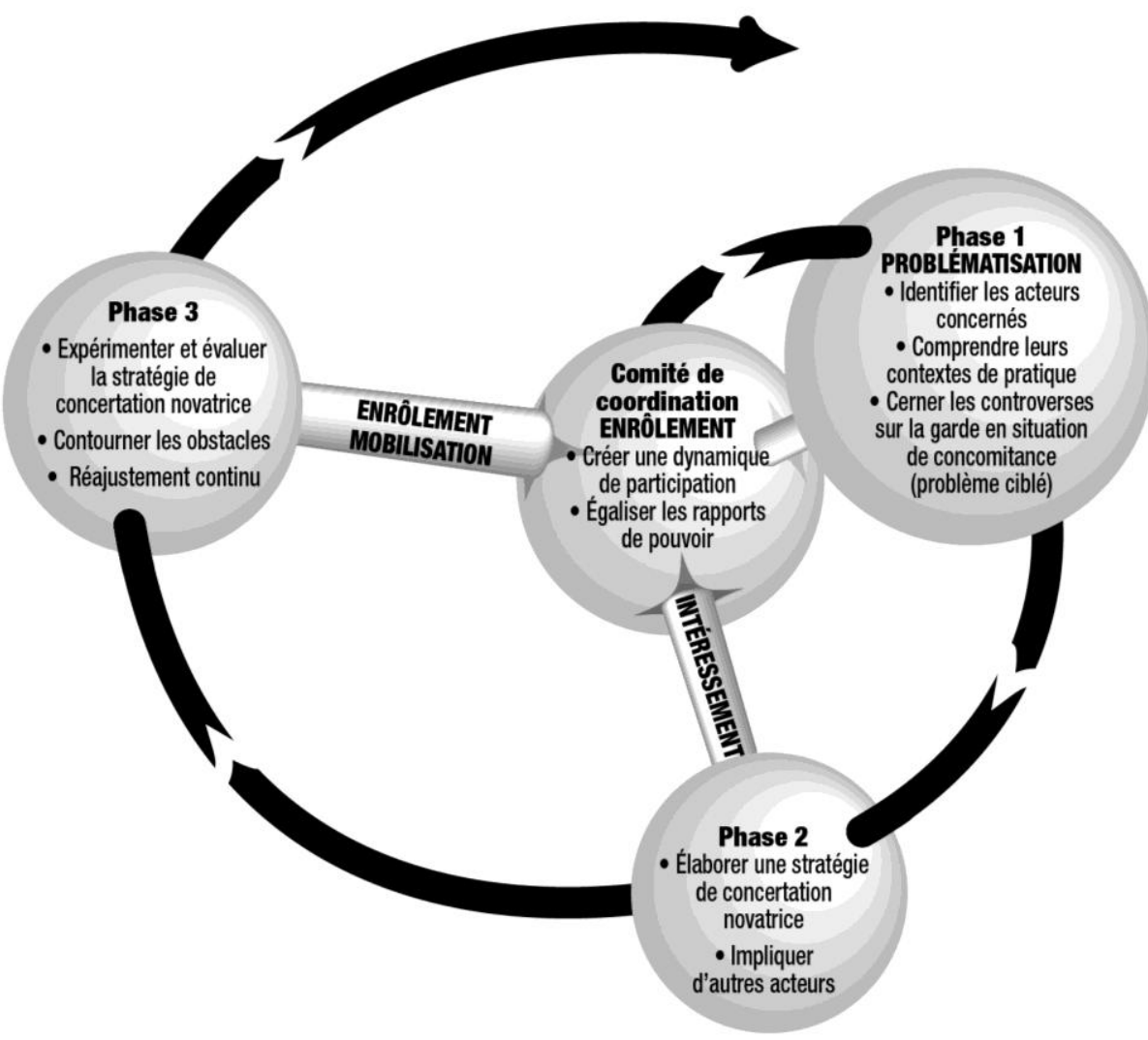

Figure 1. Illustration théorique et méthodologique du processus expérimenté dans cette étude imbriquant la sociologie de l'innovation à la démarche de recherche-action.

\section{Enrôler les membres du comité de coordination}

Comme mentionné précédemment, l'enrôlement des membres du comité de coordination a constitué un facteur déterminant dans la réalisation de cette recherche. Trois principales conditions ont émergé des analyses comme étant essentielles à l'enrôlement. Premièrement, les membres du comité ont dû s'entendre sur les concepts à la base de leur travail et mieux comprendre ce qui les distinguait et ce qui les ralliait; c'est ce qu'Amblard et al. (1996) nomment la problématisation. Les deuxième et troisième conditions sont demeurées importantes non seulement en début de projet, mais tout au long de la démarche. Il s'agit de créer une dynamique de participation suffisante et 
d'égaliser les rapports de pouvoir, conditions identifiées dans les écrits scientifiques comme essentielles dans les projets de concertation entre des partenaires provenant de divers milieux de pratique (Bilodeau et al., 2003; Lessard et al., 2006; Potito et al., 2009).

\section{Condition 1 : problématiser}

Choisir un problème concret

Dès la phase d'élaboration du projet, les milieux de pratique partenaires ont ciblé un problème concret sur lequel ils souhaitaient vivement travailler dans le cadre de la recherche-action. Parmi les diverses difficultés qu'ils rencontraient dans leur pratique d'intervention auprès des familles confrontées à la concomitance, celles qui leur ont semblé les plus névralgiques se rapportaient aux controverses entourant la garde des enfants. D'ailleurs, les écrits scientifiques renforcent la pertinence du choix de cet objet d'étude (Jaffe et al., 2005; Lapierre et al., 2004; Postmus \& Ortega, 2005). Les membres du comité constatent donc que la collaboration entre les différents organismes d'aide psychosociale est difficile dans les situations de concomitance, d'où la pertinence d'élaborer une stratégie d'intervention différente et plus concertée. «La réponse de type judiciaire est peut-être justement le symptôme de l'absence de prise en main communautaire de ce problème; la justice vient remplacer ou se substituer à des acteurs qui ne travaillent pas ensemble » (CR-juin 2004). Puisque les écrits méthodologiques sur la recherche-action indiquent que ce type de recherche doit partir d'un problème réel qui a des manifestations très concrètes dans la pratique des acteurs concernés (Mayer \& Ouellet, 1997), c'est l'approche méthodologique qui a été retenue pour la réalisation du projet. Ce choix méthodologique a contribué à l'intéressement des acteurs concernés puisque ceux-ci avaient des attentes à ce que la recherche génère des résultats concrets auxquels ils pourraient avoir accès au fur et à mesure de l'avancement du projet.

S'entendre sur une définition du concept de garde

$\mathrm{Au}$ départ, deux conceptions du concept de garde cohabitaient au sein du comité : l'une s'inspirant de la définition officielle du droit de la famille qui stipule qu'un parent qui a la garde légale d'un enfant est responsable, sur le plan légal, des décisions concernant son enfant; et l'autre définition, plus large, qui inclut les dimensions psychosociales de la pratique auprès des familles concernées. En effet, l'intervention auprès des familles en situation de concomitance inclut bien sûr le travail entourant les décisions légales concernant la garde des enfants. Mais, pour plusieurs milieux de pratique, leur travail consiste surtout à habiliter ou à responsabiliser les parents, à modifier les conditions d'exercice de la parentalité (ex. : le travail sur la dynamique de 
violence conjugale). Ces interventions sont importantes dans une perspective de soutien aux parents ou plus globalement à la famille, que ce soit dans la période précédant la décision légale ou lorsque le jugement de garde est déjà prononcé. C'est pourquoi le comité a opté pour un travail sur les controverses entourant la garde. Cette façon de problématiser a permis à chaque partenaire de percevoir plus clairement la pertinence de sa participation au projet et, par conséquent, a contribué à augmenter l'intérêt de chacun. D'ailleurs, ce lien entre problématisation et intéressement montre bien la circularité du cadre théorique tel qu'expérimenté dans le présent projet: les étapes ne sont pas réalisées de façon linéaire, mais elles se chevauchent et s'inscrivent dans une démarche en spirale avec des allers-retours entre la réflexion (collecte et analyse de données) et l'action (élaboration d'une stratégie et d'une expérimentation) (Lavoie et al., 2003).

Cerner les controverses et les convergences et comprendre les contextes dans lesquels elles se manifestent

Les controverses identifiées lors de l'analyse du contenu des groupes focalisés (phase 1) ont fait l'objet d'un autre article (Lessard et al., 2010). La première controverse concerne le maintien du lien entre le père aux comportements violents et ses enfants : dans ces situations, faut-il travailler sur les habiletés parentales du père en vue de maintenir la relation avec ses enfants? La réponse à cette question diverge selon les milieux de pratique, en fonction de leur représentation de la problématique et selon la clientèle avec laquelle ils travaillent (Lessard et al., 2010). Une seconde controverse fait état de difficultés de collaboration entre les acteurs qui travaillent en contexte volontaire et ceux dont le mandat consiste à appliquer des lois ou des ordonnances de la cour. La troisième controverse réfère aux difficultés rencontrées par les intervenants dans l'intervention auprès des familles autochtones ou immigrantes confrontées à de la violence. Le défi d'une intervention sensible à la culture de la famille aidée s'ajoute alors à la complexité de l'intervention en situation de concomitance. Malgré ces controverses, un aspect de la problématique rallie l'ensemble des groupes, soit l'importance d'assurer la sécurité des victimes, et plus particulièrement celle des enfants, dans l'intervention auprès de ces familles (Lessard et al., 2010). À chaque rencontre du comité de coordination, une situation clinique était discutée, permettant ainsi aux membres de percevoir les manifestations concrètes des divergences entre les discours et des contextes de pratique de chacun, mais aussi de constater le souci de chacun eu égard aux enjeux de sécurité et aux besoins diversifiés des membres de ces familles. 


\section{Condition 2 : créer une dynamique de participation suffisante}

La recherche-action comporte une dimension participative alliant recherche et pratique et orientée vers un changement social (Mayer \& Ouellet, 1997). Les membres du comité de coordination ont participé à toutes les décisions liées au déroulement de la recherche-action. Cette implication leur a permis de s'approprier le projet et est progressivement devenue génératrice d'un engagement de plus en plus grand. Pour certains, particulièrement ceux qui avaient moins d'expérience en recherche, une si grande participation a pu représenter un défi important à relever. "Plusieurs membres du comité de coordination en sont à leur première participation à un projet de cette envergure. Chacun de nous a pu exprimer ses inquiétudes vis-à-vis l'implication dans ce projet» (JB-fév. 2006). Si le comité a convenu de fonctionner avec souplesse, en permettant à chacun de s'impliquer selon la mesure de ses disponibilités et autres engagements, les rôles suivants ont été négociés et identifiés comme plus importants: présence aux rencontres du comité, rédaction du journal de bord après chaque rencontre, observation dans son milieu de pratique, leadership pour la mise en place des activités et pour la collecte des données dans son milieu de pratique, révision de divers documents (CR-fév. 2006). Les membres ont exprimé plus clairement leur intérêt face au projet à partir de la deuxième rencontre (CR-avril 2006).

En plus du degré d'expérience variable en recherche, les membres occupent également des fonctions différenciées au sein de leur organisation : certains sont directeurs de leur organisme alors que d'autres sont intervenants sur le terrain et redevables à au moins un supérieur dans la hiérarchie organisationnelle. Ajoutons à ces différences les cultures et les réalités bien distinctes des milieux institutionnels et communautaires. La diversité des statuts a donc soulevé la question suivante : attend-on des praticiens-chercheurs membres du comité qu'ils représentent la position de leur organisme? Le comité a convenu qu'une telle attente serait irréaliste et que chacun participerait au comité pour y apporter son expertise individuelle. Cette clarification du rôle de chacun était très importante à faire dès le démarrage du projet. Les membres ont également décidé que, si un membre devait s'absenter à une rencontre, il ne serait pas remplacé par une autre personne de son organisation. Il serait plutôt contacté individuellement pour assurer une participation continue de chaque membre aux décisions et aux étapes du projet.

Un autre aspect du fonctionnement décidé à la rencontre de février 2006, qui a probablement favorisé l'intéressement et l'appropriation du projet par les partenaires, ou qui a au moins permis une meilleure connaissance du contexte 
de pratique de chacun, a consisté à se réunir en alternance dans les différents milieux de pratique.

S'engager dans l'écriture d'un journal de bord et négocier les paramètres de l'utilisation de cet outil

Dès le début du projet, les membres du comité se sont engagés à écrire un journal de bord (JB) traitant soit du contenu des rencontres, soit du climat ou de la démarche dans laquelle se déroule la recherche-action. Le JB était acheminé par courriel à l'auxiliaire de recherche après chaque rencontre du comité de coordination. L'auxiliaire ajoutait le paragraphe de chacun à la fin du compte-rendu (CR) des rencontres en s'assurant de préserver l'anonymat de leurs propos. Au début de la démarche, la majorité des membres tenait au caractère confidentiel $\mathrm{du}$ journal de bord qui permettait «une plus grande liberté d'expression, de points de vue et d'opinions en construction » (JB-avril 2006). Toutefois, à force de partager et de débattre de leurs différents points de vue dans les rencontres du comité, la connaissance des partenaires et le sentiment de confiance étaient de plus en plus considérés comme acquis. Les membres ont donc décidé que le JB serait remplacé par une évaluation des rencontres non plus sous le couvert de l'anonymat. À la fin de chaque rencontre, chacun pourrait ainsi exprimer librement ce qu'il a aimé et moins aimé, ses attentes, déceptions, frustrations, etc. (CR-août 2008).

\section{Connaître les mandats et les contextes de chacun}

La connaissance des partenaires constitue un autre élément clé de l'implication dans un projet de concertation (Lessard et al., 2006; Wendt, 2010). Cependant, si la connaissance d'un partenaire sur le plan individuel semble s'acquérir plus rapidement, celle propre à son contexte de pratique exige plus de temps. En effet, alors que la phase d'élaboration du projet a permis aux acteurs de se familiariser avec les mandats et les contextes de chacun, la connaissance de l'autre et de son contexte est demeurée à parfaire tout au long du projet : «Plusieurs questions soulevées par les membres présents ont permis de mieux connaître le mandat de la Maison de la famille»(CR-sept. 2004); «On découvre les organismes et on s'aperçoit qu'on se connaît peu » (JB-juin 2006).

\section{Négocier les départs et les arrivées}

Au début de la phase 2, les membres ont dû revenir sur leur décision de ne pas remplacer les absents puisque l'intervenante représentant l'un des organismes partenaires du projet quittait alors son poste. Étant donné l'importance de l'implication de chacun des milieux de pratique concernés, une nouvelle intervenante représentant le même organisme a été intégrée à l'équipe, permettant ainsi de maintenir l'enrôlement de cet organisme dans le projet. Par ailleurs, pendant la phase 3 , le comité a aussi assisté à une modification du 
degré d'implication d'un autre organisme, qui s'explique par une diminution de l'intérêt en fonction de la nature des activités qui a évolué de la phase 1 à la phase 3. Cet organisme a décidé de continuer à collaborer (ex. : disponibilité pour agir à titre d'expert externe lors de discussions de cas cliniques) mais à un degré d'implication moindre que lors des étapes précédentes.

\section{Condition 3 : égaliser les rapports de pouvoir}

Un principe de base en recherche-action consiste à égaliser les rapports de pouvoir entre les chercheurs et les praticiens (Mayer \& Ouellet, 1997). Pour ce faire, les chercheurs doivent se rapprocher des milieux de pratique et se montrer sensibles aux préoccupations des intervenants sur le terrain, alors que les praticiens doivent démystifier la recherche et y participer pleinement. Les rapports de pouvoir doivent aussi être égalisés entre les milieux de pratique institutionnels et communautaires. Plusieurs stratégies peuvent favoriser des rapports plus égalitaires, autant entre les chercheurs et les praticiens qu'entre les praticiens de différentes organisations. Cependant, dans notre expérimentation, cette condition s'est avérée intimement liée à la précédente, soit la participation des membres à chacune des étapes du projet. C'est pourquoi toutes les deux se retrouvent au centre du schéma présenté à la Figure 1.

Importance de la participation de chacun à toutes les étapes et décisions

Les membres du comité de coordination ont assumé un leadership important pour la constitution et l'animation des groupes focalisés dans leur milieu de pratique (phase 1), pour l'analyse qualitative des propos recueillis dans le cadre de ces groupes, pour la préparation, l'animation et l'analyse du groupe de travail (phase 2), pour l'élaboration de la stratégie de concertation et pour l'implantation et l'évaluation de cette stratégie (phase 3). Il est à noter que certaines tâches ont suscité plus de craintes que d'autres, entre autres l'implication de chaque praticien-chercheur dans la vérification de la codification des données (processus d'accord inter-juges).

Le volet recherche (notamment par la codification) nous a ramené à l'objet et à la nature de notre groupe et au paradoxe qui, j'imagine, caractérise les recherches-actions où les partenaires sont impliqués comme chercheurs (ce qui peut être intéressant et bonifiant), mais aussi dans les tâches de la recherche (ce qui peut être un peu plus paralysant... du moins au début) (JB-décembre 2006).

Partie un peu plus complexe, celle de la codification. Ce travail ne m'apparaît pas facile pour un profane comme moi malgré les explications, j'aurai sûrement besoin d'aide... J'ai le goût et 
l'intérêt de poursuivre et de comprendre cette codification, un défi à relever (JB-décembre 2006).

Les membres ont aussi participé à la diffusion et au transfert des connaissances issues du projet par l'écriture d'articles et les présentations dans des colloques. Cette implication dans les activités de diffusion est essentielle puisque, pour générer une stratégie novatrice et intéressante pour les milieux de pratique concernés, un important travail de traduction et d'intéressement doit se faire concernant le déroulement de la recherche-action et ses résultats (Amblard et al., 1996). Les praticiens-chercheurs sont des acteurs clés dans cette démarche puisqu'ils maîtrisent d'une part le projet pour avoir participé à toutes ses étapes, et d'autre part les réalités, enjeux et intérêts propres à leur milieu de pratique respectif. Leur implication dans la diffusion et le transfert est également un exemple de leur mobilisation dans l'action. Mais pour participer pleinement, il s'avère essentiel d'être formé et informé adéquatement quant à chacune des étapes du projet et aux exigences de la démarche scientifique.

La dernière rencontre m'a apporté un éclairage nouveau sur la façon de travailler dans le domaine de la recherche, ce qui contribue à augmenter mon intérêt pour le projet et à comprendre davantage le rôle que je peux y jouer (JB-août 2006).

Les membres du comité commencent à avoir une meilleure capacité à se projeter dans les prochaines étapes du projet [...] Les rencontres du comité de coordination facilitent la compréhension et l'appropriation du langage de la recherche (CR-fév. 2007).

\section{Équilibrer la réponse aux intérêts de chaque partenaire}

Le fait d'équilibrer les rapports de pouvoir implique aussi une gestion du temps qui reconnaît et considère autant les préoccupations cliniques que celles liées à la démarche de recherche. En février 2006, plusieurs aspects liés au déroulement de la recherche ont dû être décidés collectivement. Le temps de la rencontre a été mobilisé presque uniquement pour structurer le déroulement de la recherche-action. Bien que très satisfaisant pour les acteurs du milieu universitaire qui sont partis de la rencontre avec un plan assez précis concernant le déroulement du projet, les praticiens-chercheurs sont restés sur leur appétit en ce qui a trait à la possibilité d'échanger avec des collègues d'autres milieux de pratique concernant les enjeux cliniques liés à la garde des enfants dans les situations de violence conjugale et de mauvais traitements, puisque cela constitue leurs préoccupations concrètes dans ces dossiers et l'intérêt premier de leur participation au projet. Il a donc été décidé qu'au début de chaque rencontre du comité, une période de temps serait consacrée à la discussion d'une situation clinique de violence conjugale et familiale 
comportant des enjeux liés à la garde. Ces discussions cliniques ont dès le départ été perçues comme un outil de travail productif et facilitant dans la démarche du projet. La deuxième partie de la rencontre était consacrée aux points d'information et de discussion concernant le déroulement de la recherche-action. L'équilibre dans la réponse aux besoins de chacun a permis de mettre davantage à profit la complémentarité des expertises représentées au sein du comité.

Il est certain que des échanges de ce type [discussions cliniques] peuvent influencer déjà les pratiques. Je suggère de conserver l'étude de cas en début de rencontre, car elle constitue un exercice d'apprivoisement mutuel et aide à la construction du climat de confiance (JB-avril 2006).

Je vois de mieux en mieux la complémentarité entre le volet recherche et le volet action. Les chercheurs apprennent à se frotter aux situations concrètes (et combien complexes!) de la pratique dans le cas de concomitance où il y a des enjeux liés à la garde; alors que les intervenants se familiarisent avec les différentes étapes d'un processus de recherche (JB-août 2006).

Importance du respect, de l'ouverture et du climat de confiance

Enfin, un autre facteur clé dans l'égalisation des rapports de pouvoir, d'ailleurs assez bien documenté dans les écrits (Lessard et al., 2006; Mayer \& Ouellet, 1997; Potito et al., 2009), concerne les attitudes des partenaires. Plusieurs extraits des journaux de bord des membres du comité de coordination soulignent l'importance du respect du travail de l'autre et des attitudes d'ouverture quant aux expertises et aux points de vue des autres. Ces attitudes semblent essentielles à la création d'un climat de confiance favorisant la collaboration. De plus, les grilles d'évaluation complétées par les intervenants ayant participé à la phase $3(n=24)$ montrent que ces attitudes figurent parmi les éléments les plus souvent mentionnés comme facteurs ayant facilité le déroulement des discussions de cas cliniques.

Il est à se demander si cette confiance, l'attention pour essayer de comprendre les autres points de vue et le respect mutuel ne sont pas un mélange efficace pour solutionner des controverses. Les solutions amenées ne sont pas moins complexes, mais notre préoccupation à trouver des solutions plutôt qu'à chercher à avoir raison (comme nous le rencontrons souvent dans les instances de concertation) facilite notre travail (JB-octobre 2006). 


\section{Stratégies d'intéressement d'autres acteurs}

Deux principales stratégies ont été mises de l'avant dans la perspective d'intéresser d'autres acteurs à s'engager dans la démarche : d'une part, de nombreuses activités de diffusion et de transfert des connaissances et, d'autre part, l'organisation et la réalisation d'un groupe de travail intersectoriel.

\section{Diffusion et transfert des connaissances}

Un effort de diffusion important a été investi par la présentation du projet dans de nombreux colloques (15 conférences, ateliers ou séminaires). De plus, des rencontres d'équipes et de comités ont été réalisées dans certains milieux partenaires pour la promotion du projet en vue de faciliter le déroulement de la phase 3. Des activités de transfert des connaissances portant sur les principales controverses et point de convergence identifiés dans la phase 1 ont aussi été réalisées dans la plupart des milieux de pratique avant le début de la phase 2 . Bien que ces échanges entourant les résultats de la phase 1 n'aient pas été prévus initialement dans le projet, ils ont permis aux nouveaux intervenants ayant participé à la phase 2 de connaître davantage le projet, sa démarche et ses résultats. Enfin, un séminaire de transfert des connaissances a eu lieu en janvier 2009. Il visait à discuter de la pertinence du projet, des défis d'implantation dans les milieux de pratique, en vue de sensibiliser et d'intéresser de nouveaux intervenants à s'impliquer dans la démarche. Comme mentionné précédemment, la position des praticiens-chercheurs impliqués dans le projet a facilité le transfert des connaissances dans chacun des milieux et, conséquemment, a contribué à l'intéressement d'autres acteurs.

\section{Groupe de travail intersectoriel}

La stratégie d'intéressement/enrôlement pour la phase 2 était la suivante : les chercheurs universitaires ont occupé les rôles d'animateurs pour le travail en sous-groupes et en grand groupe, alors que les praticiens-chercheurs ont exercé un rôle de leader dans le déroulement et l'orientation des discussions. Tel que recommandé par Bilodeau et al. (2003), les différents arguments ont été classifiés selon leur nature dans l'une des catégories suivantes : 1) les valeurs, 2) les savoirs d'expérience, 3 ) les savoirs scientifiques. Certains des arguments, soulevés initialement, ont été finalement rejetés après avoir été débattus avec les participants. L'analyse du déroulement des deux journées de travail a fait ressortir une certaine évolution entre la première et la seconde journée. Dans la première journée, les discussions montrent la curiosité des participants à connaître la pratique des intervenants des autres milieux (ex. : plusieurs questions de clarification ou demandes d'explication), accompagnée d'une tendance à justifier les pratiques se déroulant dans leur propre milieu de pratique. Les échanges se déroulent dans une dynamique argumentaire et de 
débat, à un niveau horizontal. Lors de la deuxième journée de travail, on remarque davantage d'échanges de nature pyramidale, c'est-à-dire que malgré les divergences entre les groupes d'intervenants, on peut percevoir un déplacement dans la position initiale des groupes qui ont maintenant plus tendance à construire sur des bases communes qu'à s'opposer et à se justifier.

Une situation clinique a été présentée et utilisée pour alimenter les discussions. Cette stratégie a d'ailleurs aussi été utilisée lors de certaines conférences ou certains ateliers de diffusion pour lesquels le public cible était constitué principalement d'intervenants sociaux. À l'automne 2007, les membres du comité de coordination ont conclu que la stratégie consistant à discuter de dossiers cliniques réels peut s'avérer pertinente pour favoriser la résolution des controverses identifiées dans la phase 1. Étant donné la très grande complexité des enjeux entourant la garde des enfants dans les situations de concomitance, cette stratégie a été retenue pour l'élaboration du modèle novateur de concertation parce qu'elle permet de tenir compte de l'unicité de chaque situation.

\section{Enrôler et mobiliser un plus grand nombre d'intervenants}

C'est à partir de la phase 3 du projet, soit à l'automne 2008, que les efforts de mobilisation dans l'action se sont étendus à un deuxième niveau d'enrôlement. Il s'agissait d'impliquer des intervenants n'ayant pas été impliqués jusqu'à présent dans la démarche. Malgré l'enrôlement et la mobilisation des praticiens-chercheurs, le recrutement des familles et des intervenants participants s'est déroulé plutôt lentement, en raison de différents obstacles.

Exemples d'enrôlement et de mobilisation des praticiens-chercheurs :

- La praticienne-chercheuse du CJQ-IU s'est engagée à animer les rencontres de discussions cliniques de la phase 3 (CR-août 2008).

- Le praticien-chercheur du Groupe d'aide aux personnes impulsives s'est engagé à organiser un séminaire de transfert des connaissances (CR-août 2008).

- La plupart des membres du comité de coordination ont participé à au moins une rencontre de discussions à titre d'experts externes au cours de la phase 3 .

\section{Obstacles rencontrés et stratégies pour maintenir la mobilisation}

À l'instar des auteurs qui ont déjà relevé cet enjeu (Banks et al., 2008; Potito et al., 2009), le manque de temps a été le principal obstacle rencontré dans ce projet. Pour élaborer le modèle de concertation et les instruments d'évaluation (phase 2), il a fallu plusieurs rencontres avant que le comité finalise, en mai 2008, l'articulation complète et opérationnelle de la stratégie (CR-mai 2008). 
«Le temps empêche souvent les discussions de se conclure sur une entente et le raisonnement est à refaire à la réunion suivante »(CR-janvier 2008); « [...] nous sommes confrontés à certaines considérations éthiques, cliniques et organisationnelles » (JB-mars 2008).

Le recrutement des participants à la phase 3 a aussi exigé beaucoup de temps. Plusieurs semaines après le début de la phase 3 , seulement

trois situations avaient été ciblées, [la première expérimentation de la stratégie de discussion clinique a eu lieu en février 2009]. Le recrutement est difficile et la sollicitation des chefs de service n'est pas aussi efficace que celle des intervenants (CR-nov. 2008).

La praticienne-chercheuse du CJQ-IU, qui exerce un rôle plus important dans le recrutement, a demandé le soutien des partenaires qui s'engageaient à identifier dans leurs clientèles les familles répondant aux critères et également suivies par le CJQ-IU à l'étape de l'évaluation-orientation du dossier (CR-nov. 2008). L'effort collectif investi par chacun par la suite pour trouver des situations répondant aux critères a porté ses fruits puisque «les familles recrutées ont été majoritairement référées par les partenaires [du CJQ-IU] » (CR-sept. 2009). Le processus d'intéressement s'est aussi poursuivi de façon continue, particulièrement dans les établissements comportant de nombreux acteurs et couvrant diverses problématiques : «Il est important de faire de nombreux rappels aux intervenants afin qu'ils gardent davantage en tête l'existence du projet» (CR-mars 2009). Les rencontres du comité de coordination sont essentielles pour permettre aux membres de s'encourager et de trouver des stratégies pour contourner les difficultés. Les membres repartent des rencontres persuadés que les efforts de chacun porteront leurs fruits ultérieurement (CR-mars 2009).

Un autre défi concerne le maintien d'un équilibre entre les investissements de chacun dans le projet et les bénéfices retirés. Cet équilibre est essentiel pour maintenir l'intérêt de tous dans le projet. Au début de la phase 3 , les membres du comité se questionnent quant au positionnement des acteurs qui participeront aux discussions cliniques à titre d'experts externes.

[Si ces experts participent aux discussions cliniques] pour transmettre leur expertise à la DPJ, cela accentuera le risque que l'expertise des organismes participants soit «utilisée » pour les décisions à prendre par la DPJ, alors qu'on souhaite plutôt un processus d'influence mutuelle entre l'ensemble des participants [...] [Tous] les intervenants participants devront saisir l'importance de ce que eux peuvent en tirer et non seulement ce qu'ils peuvent apporter aux intervenants de la DPJ (CR-nov. 2008). 
Ce questionnement s'est avéré tout à fait justifié puisqu'en juin 2009, après avoir réalisé les premières discussions cliniques de la phase 3 , le comité a constaté que :

Malheureusement, aucun autre organisme que le Centre jeunesse n'a été représenté par un intervenant qui travaillait avec la famille en question. Bien que l'activité semble bénéfique pour les intervenants du Centre jeunesse, les impacts de la discussion clinique sont peut-être plus limités alors qu'un seul organisme en profite. Il serait préférable d'avoir des intervenants travaillant avec la famille dans au moins deux organismes avec possiblement un expert qui viendrait combler le volet d'expertise non représenté si nécessaire (CR-juin 2009).

Cet extrait illustre bien l'appropriation de la démarche par les praticiens-chercheurs qui en viennent à identifier eux-mêmes les réajustements nécessaires au protocole, à créer de nouvelles solutions pour résoudre les difficultés non anticipées initialement mais qui se présentent finalement comme des obstacles à la concertation souhaitée.

Dans la même perspective, le comité a constaté que le choix du moment où se déroule la discussion clinique est particulièrement déterminant quant à l'atteinte ou non de l'objectif d'influence mutuelle de l'ensemble des intervenants participants. Il semble ainsi opportun d'éviter de tenir la discussion clinique au moment précis de la trajectoire clinique où doit être prise une décision concernant l'enfant et sa famille (ex. : lors de la révision du dossier en protection de la jeunesse). Un changement sur le moment de la rencontre a donc été effectué au protocole en cours de route (CR-fév. 2010). Enfin, le nombre d'intervenants représentant chacun des organismes partenaires influe également sur l'équilibre des expertises en présence. Le comité a donc décidé que, lorsque plus d'un intervenant du même organisme est impliqué auprès de la famille, un seul participe à la discussion clinique et agit comme intervenant pivot pour les autres intervenants de son organisme (CR-fév. 2010).

\section{Conclusion}

L'objectif de cette recherche-action était d'élaborer une stratégie de concertation favorisant la résolution des controverses entourant la garde des enfants entre les différents milieux de pratiques concernés par l'intervention psychosociale auprès des familles confrontées à de la violence conjugale et à des mauvais traitements envers les enfants. Nous avons montré dans cet article, à l'aide des concepts de la sociologie de l'innovation, comment la démarche expérimentée dans le cadre de ce projet a nécessité un travail 
d'intéressement-enrôlement-mobilisation à deux niveaux, ce qui s'est aussi avéré être une condition de succès importante. Le premier niveau renvoie à la participation des membres du comité de coordination et le deuxième niveau consiste à impliquer progressivement de plus en plus de nouveaux acteurs, notamment dans les phases 2 et 3 du projet. D'autres auteurs recommandent ce type d'approche, qui est non imposée du haut, mais dans laquelle les organismes du milieu sont parties prenantes du processus, pour la mise en place de projets de concertation (Parazelli, Hébert, Huot, Bourgon, Gélinas, Laurin, Lévesque, Rhéaume, \& Gagnon, 2003). Les résultats présentés dans cet article apportent une contribution à l'application concrète du cadre théorique retenu. La sociologie de l'innovation est ici présentée et expérimentée comme un processus circulaire comportant des allers-retours entre les phases d'intéressement, d'enrôlement et de mobilisation. L'analyse de la démarche expérimentée montre la pertinence et les forces de la recherche-action (permet de résoudre des problèmes concrets, de changer des pratiques, d'innover, d'égaliser les rapports de pouvoir) eu égard aux objectifs de l'étude. Par ailleurs, ce choix méthodologique comporte également des limites. Les deux principales nous apparaissent être le temps nécessaire à la mise en place d'une telle démarche ainsi que les défis de la transposer dans d'autres contextes semblables. L'analyse de la démarche expérimentée a été facilitée par l'utilisation des CR et des JB comme corpus de données. En effet, ces données étant colligées et validées par les membres de façon continue tout au long du projet, ce choix méthodologique s'est avéré pertinent pour analyser en profondeur la démarche expérimentée. Il est cependant important d'assurer une constance dans la façon de recueillir ces données ou d'être en mesure d'expliquer les changements opérés en cours de route, s'il y a lieu, quant au format de ces outils de collecte, surtout lorsqu'ils sont utilisés sur une longue période et par différents acteurs.

Malgré les commentaires fort positifs des intervenants qui ont participé à la phase 3, il nous apparaît essentiel de poursuivre l'expérimentation et l'évaluation de la stratégie de concertation élaborée auprès d'un plus grand nombre de familles. Le principe de réajustement continu de la stratégie en fonction des résultats de l'expérimentation devrait aussi être conservé, toujours dans l'objectif de maintenir la mobilisation des acteurs et d'intéresser de nouveaux intervenants au projet. Cet aller-retour entre recherche et pratique, entre réflexion et action, constitue à notre avis un élément clé de toute démarche visant à élaborer une stratégie novatrice pour aider des familles qui vivent des réalités complexes ainsi que pour soutenir la pratique des intervenants qui leur viennent en aide. 
L'appropriation du projet et de sa démarche par les membres du comité de coordination et leur aisance acquise progressivement par rapport au fait de devoir contourner des obstacles constituent des forces importantes sur lesquelles pourront s'appuyer les travaux futurs :

Je vois ça comme l'évolution des contraintes, on tente de voir les meilleures façons de faire dans le meilleur intérêt. Nous ne sommes pas figés dans le modèle que nous avions décidé au départ (CR-fév. 2010).

C'est comme si nous revivions les 3 premières phases mais en accéléré puisque nous savons à quoi nous attendre. Nous verrons réellement quels sont les résultats. Nous serons moins «en processus ». Il faut que notre base soit solide, je réalise ça (CR-avril 2010).

Si cette démarche a été des plus enrichissantes pour ceux et celles qui y ont pris part, il reste encore beaucoup de travail à accomplir pour améliorer la continuité et la cohérence de l'aide offerte aux familles concernées et pour répondre aux nouvelles questions qui émergent de cette recherche-action. Voici quelques exemples de ces questions qui nous animent et auxquelles les recherches futures pourraient s'attaquer: Le processus de résolution des controverses élaboré dans le cadre de cette recherche-action est-il transférable et applicable dans d'autres régions? Quelles seraient, s'il y a lieu, les caractéristiques des situations pour lesquelles l'application de la stratégie de concertation serait contre-indiquée? Lorsque la stratégie est appliquée à un plus grand nombre de familles, quels sont les résultats en ce qui a trait à la qualité, à la cohérence et à l'intégration de l'aide offerte aux familles selon les acteurs concernés (enfants, parents et intervenants)? Est-ce que les résultats eu égard à la concertation diffèrent selon les caractéristiques des intervenants participants (ex. : organisme d'appartenance, expérience de concertation antérieure) ou des familles (ex. : nature et sévérité des formes de violence)?

\section{Note}

1 Cet article a été produit grâce à l'appui financier du Centre de recherche interdisciplinaire sur la violence familiale et la violence faite aux femmes (CRI-VIFF). Nous remercions également le Fonds québécois de la recherche sur la société et la culture (FQRSC) ainsi que l'Agence de la santé et des services sociaux de la CapitaleNationale pour le financement de cette recherche-action. 


\section{Références}

Amblard, H., Bernoux, P., Herreros, G., \& Livian, Y.- F. (1996). Les nouvelles approches sociologiques des organisations. Paris : Éditions du Seuil.

Appel, A. E., \& Holden, G. W. (1998). The co-occurrence of spouse and physical child abuse: a review and appraisal. Journal of Family Psychology, 12(4), 578-599.

Banks, D., Dutch, N., \& Wang, K. (2008). Collaborative efforts to improve system response to families who are experiencing child maltreatment and domestic violence. Journal of Interpersonal Violence, 23(7), 876-902.

Banks, D., Hazen, A. L., Coben, J. H., Wang, K., \& Griffith, J. D. (2009). Collaboration between child welfare agencies and domestic violence service providers : relationship with child welfare policies and practices for addressing domestic violence. Children and Youth Services Review, 31(5), 497-505.

Beeman, S. K., Hagemeister, A. K., \& Edleson, J. L. (1999). Child protection and battered women's services: from conflict to collaboration. Child Maltreatment, 4(2), 116-126.

Bilodeau, A., Lapierre, S., \& Marchand, Y. (2003). Le partenariat : comment ça marche? Mieux s'outiller pour réussir. Régie régionale de la santé et des services sociaux de Montréal-Centre, Direction de santé publique.

Douglas, H., \& Walsh, T. (2010). Mothers, domestic violence, and child protection: toward collaboration and engagement. Violence Against Women, 16(5), 537-542.

Goddard, C., \& Bedi, G. (2010). Intimate partner violence and child abuse : a child-centred perspective. Child Abuse Review, 19(1), 5-20.

Hartley, C. C. (2002). The co-occurrence of child maltreatment and domestic violence: examining both neglect and child physical abuse. Child Maltreatment, 7(4), 349-358.

Jaffe, P., Lemmon, N., \& Poisson, S. (2005). Child custody and domestic violence: a call for safety and accountability. Thousand Oaks : Sage.

Jaffe, P. G., Crooks, C. V., \& Poisson, E. E. (2003). Common misconceptions in addressing domestic violence in child custody disputes. Juvenile \& Family Court Journal, 54(4), 57-67. 
Lapierre, S., Lessard, G., \& Turcotte, D. (2004). La séparation dans un contexte de violence conjugale : quels sont les défis pour l'intervention auprès des enfants. Dans M.- C. Saint-Jacques, D. Turcotte, S. Drapeau, \& R. Cloutier (Éds), Séparation, monoparentalité et recomposition familiale : bilan d'une réalité complexe et pistes d'actions (pp. 195-216). Québec: Presses de l'Université Laval.

Lavergne, C., Chamberland, C., Laporte, L., \& Baraldi, R. (2003). Violence conjugale: impliquer les pères et aider les mères pour protéger les enfants. Centre d'excellence pour la protection et le bien-être des enfants.

Lavoie. L., Marquis, D., \& Laurin, P. (2003). La recherche-action : théorie et pratique. Manuel d'autoformation. Québec: Presses de l'Université du Québec.

Lessard, G., Flynn, C., Turcotte, P., Damant, D., Vézina, J.- F., Godin, M.- F., Paradis, F., Delisle, R., Alcedo, Y., Juneau, L., Rock, L., \& Rondeau-Cantin, S. (2010). Child custody issues and co-occurrence of intimate partner violence and child maltreatment : controversies and points of agreement amongst practitioners. Child \& Family Social Work, 15(4), 492-500.

Lessard, G., Lavergne, C., Chamberland, C., Damant, D., \& Turcotte, D. (2006). Conditions for resolving controversies between social actors in domestic violence and youth protection services: toward innovative collaborative practices. Children and Youth Services Review, 28(5), 511-534.

Malik, N. M., Silverman, J., Wang, K., \& Janczewski, C. (2008a). Domestic violence and dependency courts : the greenbook demonstration experience. Journal of Interpersonal Violence, 23(7), 956-980.

Malik, N. M., Ward, K., \& Janczewski, C. (2008b). Coordinated community response to family violence: the role of domestic violence service organizations. Journal of Interpersonal Violence, 23(7), 933-955.

Margolin, G., Vickerman, K. A., Ramos, M. C., Serrano, S. D., Gordis, E. B., Iturralde, E., Oliver, P. H., \& Spies, L. A. (2009). Youth exposed to violence : stability, co-occurrence, and context. Clinical Child and Family Psychology Review, 12(1), 39-54. 
Mayer, R. \& Deslauriers, J.- P. (2000). Quelques éléments d'analyse qualitative : l'analyse de contenu, l'analyse ancrée, l'induction analytique et le récit de vie. Dans R. Mayer, F. Ouellet, M.- C. Saint-Jacques, D. Turcotte (Éds), Méthodes de recherche en intervention sociale. Montréal : Gaëtan Morin.

Mayer, R. \& Ouellet, F. (1997). La diversité des approches dans les recherches qualitatives au Québec depuis 1970 : le cas du champ des services de santé et des services sociaux. Dans J. Poupart, L.- H. Groulx, R. Mayer, J.- P. Deslauriers, A. Lapierre, \& A. P. Pires (Éds), La recherche qualitative : diversité des champs et des pratiques au Québec. Boucherville: Gaëtan Morin.

Osofsky, J. D. (2003). Prevalence of children's exposure to domestic violence and child maltreatment: implications for prevention and intervention. Clinical Child and Family Psychology Review, 6(3), 161-170.

Parazelli, M., Hébert, J., Huot, F., Bourgon, M., Gélinas, C., Laurin, C., Lévesque, S., Rhéaume, M., \& Gagnon, S. (2003). Les programmes de prévention précoce: fondements théoriques et pièges démocratiques. Service social, 50(1), 81-121.

Postmus, J. L., \& Ortega, D. (2005). Serving two masters : when domestic violence and child abuse overlap. Families in Society, 86(4), 483-490.

Potito, C., Day, A., Carson, E., \& O’Leary, P. (2009). Domestic violence and child protection : partnerships and collaboration. Australian Social Work, 62(3), 369-387.

Reason, P. \& Bradbury, H. (2001). Introduction : inquiry and participation in search of a world worthy of human aspiration. Dans P. Reason \& H. Bradbury (Éds), Handbook of action research. London : Sage.

Rinfret-Raynor, M., Dubé, M., Drouin, C., Maillé, N., \& Harper, E. (2008). Violence conjugale post-séparation en contexte d'exercice des droits d'accès aux enfants. Dans S. Arcand, D. Damant, S. Gravel, \& E. Harper (Éds), Violences faites aux femmes. Québec: Presses de l'Université du Québec.

Shipman, K. L., Rossman, B. B. R., \& West, J. C. (1999). Co-occurrence of spousal violence and child abuse: conceptual implications. Child Maltreatment, 4(2), 93-102. 
Tutty, L. (2007, Mai). Supervised visitation and exchange centres for domestic violence : an environmental scan of Canadian programs. Communication présentée à la troisième Conférence internationale sur les enfants exposés à la violence conjugale. London, $\mathrm{ON}$.

Wendt, S. (2010). Building and sustaining local co-ordination : an Australian rural community responds to domestic and family violence. British Journal of Social Work, 40(1), 44-62.

Geneviève Lessard, Ph.D., T.S., est professeure agrégée à l'École de service social de l'Université Laval et directrice du Centre de recherche interdisciplinaire sur la violence familiale et la violence faite aux femmes (CRI_VIFF). Ses travaux portent sur les diverses formes de victimisation subies par les enfants et les adolescents, notamment l'exposition à la violence conjugale ainsi que sur la concertation entre les ressources d'aide concernées par la violence conjugale et familiale.

Catherine Flynn, M. Serv.Soc., a obtenu la prestigieuse bourse Vanier pour la réalisation de son doctorat (en cours) en service social à l'Université de Montréal. Auxiliaire de recherche au CRI-VIFF depuis 2006, ses travaux portent sur l'expérience et les besoins des femmes autochtones en milieu urbain et victimes de violence conjugale ainsi que sur les stratégies développées par les enfants autochtones et immigrés en réponse à la violence à caractère sexiste et raciale.

Pierre Turcotte, Ph.D., T.S., est professeur agrégé et directeur de l'École de service social de l'Université Laval. Il enseigne l'intervention auprès des individus et des familles. Ses recherches portent sur l'intervention auprès des conjoints aux comportements violents en contexte conjugal ou familial et la socialisation de genre. Il a d'abord été intervenant auprès de cette clientèle pendant près de 15 ans.

Lorraine Juneau, bachelière en service social, est réviseure au Centre jeunesse de Québec - Institut universitaire (CJQ-IU). Intervenante en protection de la jeunesse pendant de nombreuses années, elle a développé une expertise dans l'intervention individuelle et de groupe auprès des familles confrontées à de la violence et a participé à des tables de réflexions cliniques visant le développement des pratiques en Centre jeunesse dans les situations de violence familiale.

Rhéa Delisle, T.S., est intervenante depuis près de 20 ans au Centre de santé et de services sociaux de la Vieille-Capitale au sein de l'équipe Famille-Enfance-Jeunesse. Responsable du programme de groupe Ensemble on découvre et coauteure du guide accompagnant ce programme, elle a développé une expertise reconnue sur la problématique et l'intervention auprès des enfants exposés à la violence conjugale. Elle offre également diverses formations et conférences dans ce domaine. 
Valérie Meunier, B.Ps., est directrice du Groupe d'aide aux personnes impulsives, organisme au sein duquel elle travaille depuis 1998 auprès d'une clientèle d'hommes ayant un problème de violence conjugale et familiale. Elle a aussi œuvré pendant cinq ans en maison d'hébergement auprès de femmes victimes de violence et de leurs enfants. Elle siège au conseil d'administration de l'association provinciale À cour d'homme et de la Maison des femmes de Québec.

Marie-France Godin, directrice générale de la maison de la Famille DVS (Droits de visite et de sortie), collabore étroitement avec la Cour Supérieure (Chambre de la famille) et la Cour du Québec (Chambre de la jeunesse). Elle est également, depuis octobre 2006, membre fondateur et administratrice du Regroupement québécois des ressources de supervision de droits d'accès. Elle porte un intérêt marqué pour la famille, la protection de l'enfance et les enjeux reliés à la garde.

Yennelys Alcedo, intervenante jeunesse à la Maison pour femmes immigrantes depuis 2001, est responsable du Programme mères-enfants au sein de cet organisme. Ses domaines d'expertises sont la violence faite aux femmes et aux enfants en milieu familial, la revendication pour les droits des femmes et l'approche multiculturelle. Avec une riche expérience à son actif, madame Alcedo a obtenu des certificats en publicité et marketing dans son pays d'origine (Venezuela).

Annie Dumont, T.S., est étudiante à la maîtrise en service social à l'Université Laval, auxiliaire de recherche au CRI-VIFF et intervenante au Centre d'aide et de lutte contre les agressions à caractère sexuel (CALACS) de Québec. Détentrice de plusieurs années d'expérience en maison d'hébergement pour femmes et enfants victimes de violence conjugale, ses intérêts de recherche portent sur la polyvictimisation en tant que variable modératrice des conséquences de l'exposition à la violence conjugale sur les jeunes. 